\title{
X-RAY DETECTION OF THE NUCLEAR SOURCE IN THE CYGNUS A GALAXY
}

\author{
D.E. HARRIS \\ Center for Astrophysics, Cambridge, MA, USA \\ R.A. PERLEY \\ National Radio Astronomy Observatory, Socorro, NM, USA \\ and \\ C.L. CARILLI \\ Sterrewacht te Leiden, Leiden, the Netherlands
}

\begin{abstract}
From a ROSAT HRI observation of Cygnus A (42 ksec), we detect x-ray emission from the galaxy identified with the radio source. This was accomplished by subtracting a modified King model: (Surface brightness $\propto$ to $\left[1+(r / a)^{2}\right]^{(0.5-3 \beta)}$ ) in order to study residual features once the main body of emission from diffuse gas had been deleted. The central source was present for all acceptable values of the core radius, $a$, and exponent, $\beta$. Details of the image processing, an evaluation of emission from the radio hotspots, and a study of the effect of the radio lobes on the gas distribution will be presented elsewhere.
\end{abstract}

Key words: X-ray Emission

Although an unresolved $x$-ray source coincident with the optical galaxy has not heretofore been detected directly, Arnaud et al. (1987) made a strong case for a power-law component in their spectral analysis of data from several missions. To explain the failure to detect the core source with the Einstein HRI, they hypothesized a column density intrinsic to the source of $\mathrm{N}_{H}=8.2 \times 10^{22} \mathrm{~cm}^{-2}$, a number which corresponds to 50 magnitudes of optical extinction, $A_{v}$, (using $N_{H}$ $=4.8 \times 10^{21} \times \mathrm{E}(\mathrm{B}-\mathrm{V})$ and $\left.\mathrm{A}_{\mathrm{v}}=3.1 \mathrm{E}(\mathrm{B}-\mathrm{V})\right)$. Obviously the only way to accommodate this amount of absorption would be to site the emission at the center of the galaxy.

Only a rough estimate of the intensity can be obtained because it is difficult to make a correction for the background and for obvious reasons, the residual intensity is dependent on the amplitude of the subtracted King model. For King models normalized from $87 \%$ to $100 \%$ of the counts from the cluster gas within $r=125$ ", the intensity of the core ranges from 272 to 184 counts. After an $18 \%$ correction for scattering of photons beyond the measuring area, we thus assign a value of $270+/-60$ counts to the central source. Most of these can be represented by an unresolved component, but there appears to be some extended emission present in the measuring circle of radius 10 ".

Transforming the countrate to flux and luminosity presents another large uncertainty because of the unknown amount of absorption. For no intrinsic absorption (i.e. $\mathrm{N}_{H}=3.3 \times 10^{21} \mathrm{~cm}^{-2}$, the galactic value) our countrate would be consistent

T. J.-L. Courvoisier and A. Blecha: Multi-Wavelength Continuum Emission of AGN, 375-376.

(C) 1994 IAU. Printed in the Netherlands. 
with a $2-10 \mathrm{keV}$ luminosity of $3.8 \times 10^{42} \mathrm{erg} / \mathrm{s}$. For an additional $8.2 \times 10^{22} \mathrm{~cm}^{-2}$ at the source, a $2-10 \mathrm{keV}$ luminosity of $1.6 \times 10^{45} \mathrm{erg} / \mathrm{s}$ would be required. Adjusting the intrinsic column density to obtain the Arnaud et al. (1987) luminosity (converted to $\mathrm{H}_{o}=75 \mathrm{~km} / \mathrm{s} / \mathrm{Mpc}$ ) of $2.3 \times 10^{44} \mathrm{erg} / \mathrm{s}$ requires $\mathrm{N}_{H}$ (at the source) = $(4.6 \pm 0.4) \times 10^{22} \mathrm{~cm}^{-2}$, which corresponds to $A_{v}=29.7$ magnitudes. Although our data provide no constraints on the amount of absorption if the luminosity is not known, it is obvious that for agreement with Arnaud's spectrum, either the absorption is significantly less than $8.2 \times 10^{22}$ or the source is 7 times more luminous than it was ten years ago. Since both possibilities appear plausible, we searched for corroborating evidence.

If the core source is heavily absorbed, only the highest energy photons within the ROSAT HRI band will survive the natural highpass filter provided by the ISM of Cyg A. For $\mathrm{N}_{H}=5 \times 10^{22} \mathrm{~cm}^{-2}$, the transmission at $2 \mathrm{keV}$ is $12 \%$. We examined the Pulse Height distributions of counts for the core source (circles with $r=5$ " and $r=10 ")$ and compared them to the distribution in an annulus from 15" to 25 ". The expected signature for significant absorption is present in the data at very low significance $(<1 \sigma)$. The median channel number (i.e. half the counts in higher channels, half in lower) is marginally higher for the source than for the "background" annulus.

We searched for variability by examining the data from 3 epochs (Nov91, Dec91, and May92). The count rate for each data segment was measured for circles centered on the emission peak with radii of $2.5,5,7.5$, and 10". As the area of the measuring circle increases, there is more dilution from the cluster gas but for small circles, aspect jitter means that many counts from the core source will be outside the circle. There is no convincing evidence (i.e. $>2 \sigma$ ) for variation greater than $20 \%$ on a 1 to 6 month timescale.

Both of these tests are inconclusive, partly because of the difficulty of isolating the counts from the core source from those of the cluster gas in the same direction. While our data have adequate spatial resolution to demonstrate the reality of the core source, a substantial improvement in spectral resolution and a longer time base will be required to answer these questions.

\section{References}

Arnaud, K.A., Johnston, R.M., Fabian, A.C., Crawford, C.S., Nulsen, P.E., Shafer, R.A., and Mushotzky, R.F. 1987, M.N.R.A.S., 227, 241 